\title{
Optimal aerodynamic control by matched asymptotic expansions ${ }^{\dagger}$
}

\author{
FRED FROSTIC $\ddagger$ AND NGUYEN $X$. VINH $\S$
}

Department of Aerospace Engineering, The University of Michigan, Ann Arbor, MI 48104, U.S.A.

(Received 3 November 1975)

\begin{abstract}
This paper presents the solution for the optimal lift and bank modulation of a reentry vehicle using the method of matched asymptotic expansions. The solution, which contains all effects of aerodynamic, gravitational, and centrifugal forces, is uniformly valid everywhere.

The solution for the outer region, where gravitational force is predominant is obtained in closed form. To date, for the inner region, only four of the five adjoint equations for the free time problem have been integrated in closed form. The fifth equation has been integrated by considering the free range problem, or by using a linear approximation of the drag polar near the optimal lift control. By a new invariant relation, and a transformation of the fifth adjoint variable, it will be shown in this paper that, for the most general drag polar, the fifth equation can be integrated by quadrature. The equation will be completely integrated for a generalized drag polar. The inner solution will be matched with the outer solution providing a composite solution uniformly valid everywhere.
\end{abstract}

\section{Introduction}

THIS PAPER presents the solution for the optimal lift and bank modulation of an entry vehicle using the method of matched asymptotic expansions. The solution, which contains all effects of aerodynamic, gravitational, and centrifugal forces, is uniformly valid everywhere.

\section{Equations of motion}

The equations of motion of a non-thrusting, lifting vehicle entering a planetary atmosphere, assumed to be at rest around a spherical, non rotating planet are (Fig. 1)

$$
\begin{aligned}
\frac{\mathrm{d} r}{\mathrm{~d} t} & =V \sin \gamma \\
\frac{\mathrm{d} \theta}{\mathrm{d} t} & =\frac{V \cos \gamma \cos \psi}{r \cos \phi} \\
\frac{\mathrm{d} \phi}{\mathrm{d} t} & =\frac{V \cos \gamma \sin \psi}{r} \\
\frac{\mathrm{d} V}{\mathrm{~d} t} & =-\frac{\rho S C_{D} V^{2}}{2 m}-g \sin \gamma \\
V \frac{\mathrm{d} \gamma}{\mathrm{d} t} & =\frac{\rho S C_{L} V^{2}}{2 m} \cos \sigma-\left(g-\frac{V^{2}}{r}\right) \cos \gamma \\
V \frac{\mathrm{d} \psi}{\mathrm{d} t} & =\frac{\rho S C_{L} V^{2}}{2 m \cos \gamma} \sin \sigma-\frac{V^{2}}{r} \cos \gamma \cos \psi \tan \phi .
\end{aligned}
$$

†Paper presented at the XXVIth International Astronautical Congress of the International Astronautical Federation, Lisbon, Portugal, 21-27 September 1975.

$\ddagger$ Major, USAF.

\$Professor. 


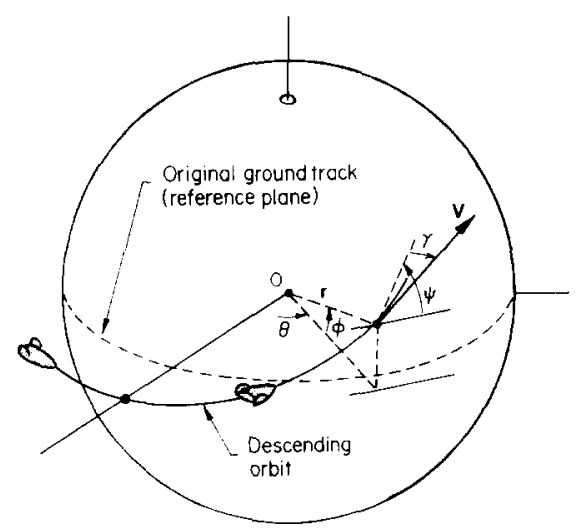

Fig. 1. Coordinates system.

They are the equations governing the variations of the six variables $r, \theta, \phi, V, \gamma$, and $\psi$ defining the state of the vehicle, considered as a point with constant mass $m$. The flight path angle $\gamma$ is defined positive above the local horizontal plane. The initial plane is taken as plane of reference which we shall refer to as the equatorial plane. The heading angle $\psi$ is then positive toward the North pole, and the bank angle $\sigma$ is selected such that for positive $\sigma$ the vehicle is turning to the left. This bank angle is defined as the angle between the local vertical plane containing the velocity, that is the $(r, V)$ plane, and the plane containing the aerodynamic force $A$ and the velocity $V$, the $(A, V)$ plane.

The gravitational field is a Newtonian field, and we have

$$
g(r)=\frac{g_{s} r_{s}^{2}}{r^{2}}
$$

where subscript $s$ denotes a reference level, taken as sea level.

It is adequate to use an exponential atmosphere

$$
\rho=\rho_{s} \mathrm{e}^{-\beta y}
$$

where $y$ is the altitude and $\beta$ a constant.

The aerodynamic force is controlled through the bank angle $\sigma$ and the lift coefficient $C_{L}$. It is assumed that there exists a lift-drag relationship for each flight aerodynamic configuration. We shall use as lift control a rescaled lift coefficient $\lambda$ defined as

$$
C_{\mathrm{L}}=C_{\mathrm{L}}^{*} \lambda
$$

where $C_{L}^{*}$ is the lift coefficient corresponding to the maximum lift-to-drag ratio. In this way, when $\lambda=1$, the flight is effected at maximum lift-to-drag ratio. For the drag coefficient we have

$$
C_{D}=C_{D}^{*} f(\lambda)
$$


where $C_{D}^{*}$ is the drag coefficient corresponding to maximum lift-to-drag ratio and $f(\lambda)$ is some function describing the drag polar.

The controls $\sigma$ and $\lambda$ are assumed to be contained in the set

$$
\begin{aligned}
& 0 \leqslant|\lambda| \leqslant \lambda_{\max } \\
& 0 \leqslant|\sigma| \leqslant \sigma_{\max } .
\end{aligned}
$$

For free time problems, it is convenient to use the first of the eqns (1) to eliminate $t$. We have

$$
\begin{aligned}
\frac{\mathrm{d} \theta}{\mathrm{d} r} & =\frac{\cos \psi}{r \cos \phi \tan \gamma} \\
\frac{\mathrm{d} \phi}{\mathrm{d} r} & =\frac{\sin \psi}{r \tan \gamma} \\
\frac{\mathrm{d} V^{2}}{\mathrm{~d} r} & =-\frac{\rho S C_{D} V^{2}}{m \sin \gamma}-2 g \\
\frac{\mathrm{d} \gamma}{\mathrm{d} r} & =\frac{\rho S C_{L} \cos \sigma}{2 m \sin \gamma}-\left(\frac{g}{V^{2}}-\frac{1}{r}\right) \frac{\cos \gamma}{\sin \gamma} \\
\frac{\mathrm{d} \psi}{\mathrm{d} r} & =\frac{\rho S C_{L} \sin \sigma}{2 m \sin \gamma \cos \gamma}-\frac{\cos \psi \tan \phi}{r \tan \gamma} .
\end{aligned}
$$

We define the following dimensionless quantities

$$
\begin{gathered}
h=\frac{y}{r_{s}}, \quad \frac{r}{r_{s}}=1+h, \quad \frac{g}{g_{s}}=\frac{1}{(1+h)^{2}} \\
u=\frac{V^{2}}{g_{s} r_{s}}, \quad B=\frac{\rho_{s} S C C_{L}^{*}}{2 m \beta}, \quad E^{*}=\frac{C_{L}^{*}}{C_{D}^{*}}, \quad \epsilon=\frac{1}{\beta r_{s}} .
\end{gathered}
$$

Upon using the eqns (3)-(5) and (8) into eqns (7), we have the dimensionless equations of motion with the dimensionless altitude $h$ used as independent variable.

$$
\begin{aligned}
& \frac{\mathrm{d} \theta}{\mathrm{d} h}=\frac{\cos \psi \cot \gamma}{(1+h) \cos \phi} \\
& \frac{\mathrm{d} \phi}{\mathrm{d} h}=\frac{\sin \psi \cot \gamma}{(1+h)} \\
& \frac{\mathrm{d} \psi}{\mathrm{d} h}=\frac{B \lambda \sin \sigma \mathrm{e}^{-h / \epsilon}}{\epsilon \sin \gamma \cos \gamma}-\frac{\cos \psi \tan \phi \cot \gamma}{(1+h)} \\
& \frac{\mathrm{d} u}{\mathrm{~d} h}=-\frac{2 B f(\lambda) u \mathrm{e}^{-h / \epsilon}}{\epsilon E^{*} \sin \gamma}-\frac{2}{(1+h)^{2}} \\
& \frac{\mathrm{d} \gamma}{\mathrm{d} h}=\frac{B \lambda \cos \sigma \mathrm{e}^{-h / \epsilon}}{\epsilon \sin \gamma}+\left[\frac{1}{(1+h)}-\frac{1}{u(1+h)^{2}}\right] \cot \gamma
\end{aligned}
$$

Since $\epsilon=1 / \beta r$, is a small quantity, e.g. for the Earth's atmosphere $\epsilon=1 / 900$, 
the set of eqn (9) is very convenient for an analytical integration using an appropriate perturbations theory. For a variational problem this set is coupled with the set of adjoint equations of the same order making the solution inextricably difficult. Fortunately, for optimization of an entry trajectory, since at high altitude the solution is nearly Keplerian, method of directly matched asymptotic expansions is available. It has been used to solve three dimensional entry at constant bank and lift coefficient (Busemann et al., 1975), or simple optimization problem (Shi, 1971). We shall apply this method, proven to be accurate, in finding general optimum control laws for the lift coefficient and the bank angle without any simplification to any restricted class of trajectories except that the time of flight is free. Since the motion is nearly Keplerian at high altitude, we shall use a new set of variables $(\alpha, \Omega, I)$ to replace the set of variables $(\theta, \phi, \psi)$. They are related by the equations

$$
\begin{gathered}
\cos I=\cos \phi \cos \psi \\
\sin \phi=\sin I \sin \alpha \\
\cos \alpha=\cos \phi \cos (\theta-\Omega) .
\end{gathered}
$$

From Fig. 2, it is seen that $I$ is the inclination of the plane of the osculating orbit and $\Omega$ is the longitude of the ascending node. The angle $\alpha$ is the angle between the line of the ascending node and the position vector. Using the transformation (10) into eqn (9) we have the final form

$$
\begin{gathered}
\frac{\mathrm{d} \alpha}{\mathrm{d} h}=-\frac{\sin \alpha}{\tan I}\left(\frac{B \lambda \sin \sigma \mathrm{e}^{-h / \epsilon}}{\epsilon \sin \gamma \cos \gamma}\right)+\frac{\cot \gamma}{(1+h)} \\
\frac{\mathrm{d} \Omega}{\mathrm{d} h}=\frac{\sin \alpha}{\sin I}\left(\frac{B \lambda \sin \sigma \mathrm{e}^{-h / \epsilon}}{\epsilon \sin \gamma \cos \gamma}\right)
\end{gathered}
$$

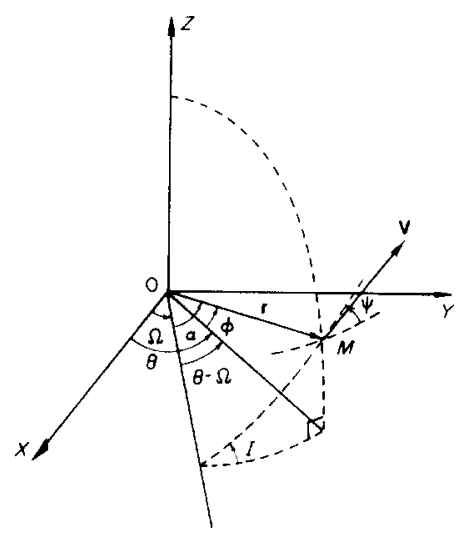

Fig. 2. The osculating plane and the elements of the orbit. 


$$
\begin{aligned}
& \frac{\mathrm{d} I}{\mathrm{~d} h}=\cos \alpha\left(\frac{B \lambda \sin \sigma \mathrm{e}^{-h / \epsilon}}{\epsilon \sin \gamma \cos \gamma}\right) \\
& \frac{\mathrm{d} u}{\mathrm{~d} h}=-\frac{2 B f(\lambda) u \mathrm{e}^{-h / \epsilon}}{\epsilon E^{*} \sin \gamma}-\frac{2}{(1+h)^{2}} \\
& \frac{\mathrm{d} \gamma}{\mathrm{d} h}=\frac{B \lambda \cos \sigma \mathrm{e}^{-h / \epsilon}}{\epsilon \sin \gamma}+\left[\frac{1}{(1+h)}-\frac{1}{u(1+h)^{2}}\right] \cot \gamma
\end{aligned}
$$

\section{Optimal control}

The state equations, eqns (11) are most suitable for an integration using the method of matched asymptotic expansions. Following the maximum principle, to have the optimum control we introduce the adjoint vector $\mathrm{p}=\left(p_{\alpha}, p_{\Omega}, p_{i}, p_{u}, p_{y}\right)$ to form the Hamiltonian.

$$
\begin{aligned}
H=\frac{B \lambda \sin \sigma \mathrm{e}^{-h / \epsilon}}{\epsilon \sin \gamma \cos \gamma}\left[-p_{\alpha} \frac{\sin \alpha}{\tan I}+p_{\Omega} \frac{\sin \alpha}{\sin I}+p_{I} \cos \alpha\right] \\
+p_{\alpha} \frac{\cot \gamma}{1+h}-\frac{2 p_{u} B f(\lambda) u \mathrm{e}^{-h / \epsilon}}{\epsilon E^{*} \sin \gamma}-\frac{2 p_{u}}{(1+h)^{2}} \\
+\frac{p_{\gamma} B \lambda \cos \sigma \mathrm{e}^{-h / \epsilon}}{\epsilon \sin \gamma}+p_{\gamma}\left[\frac{1}{1+h}-\frac{1}{u(1+h)^{2}}\right] \cot \gamma .
\end{aligned}
$$

To minimize any performance index, at each instant we maximize $H$ with respect to $\lambda$ and $\sigma$. The lift control is either $|\lambda|=\lambda_{\max }$ or a variable $\lambda$ such that $\mathrm{d} H / \mathrm{d} \lambda=0$. For variable lift control we have

$$
\frac{\mathrm{d} f}{\mathrm{~d} \lambda}=\frac{E^{*}}{2 p_{u} u}\left[\left(-p_{\alpha} \frac{\sin \alpha}{\tan I}+p_{\mathrm{\Omega}} \frac{\sin \alpha}{\sin I}+p_{I} \cos \alpha\right) \frac{\sin \sigma}{\cos \gamma}+p_{\gamma} \cos \sigma\right]
$$

The bank control is either $|\sigma|=\sigma_{\max }$ or a variable $\sigma$ such that $\mathrm{d} H / \mathrm{d} \sigma=0$. For variable bank control we have

$$
\tan \sigma=\frac{1}{p_{\gamma} \cos \gamma}\left[-p_{\alpha} \frac{\sin \alpha}{\tan I}+p_{\Omega} \frac{\sin \alpha}{\sin I}+p_{I} \cos \alpha\right]
$$

In particular, when the vehicle is flown with both variable lift and bank control we can combine the equations to obtain

$$
\frac{\mathrm{d} f}{\mathrm{~d} \lambda} \sin \sigma=\frac{E^{*}}{2 p_{u} u \cos \gamma}\left(-p_{\alpha} \frac{\sin \alpha}{\tan I}+p_{\Omega} \frac{\sin \alpha}{\sin I}+p_{I} \cos \alpha\right)
$$

and

$$
\frac{\mathrm{d} f}{\mathrm{~d} \lambda} \cos \sigma=\frac{E^{*} p_{\gamma}}{2 p_{u} u}
$$

The optimal control laws are independent of the vehicle characteristic 
coefficient $B$, as defined in eqn (8). Hence the result obtained is valid for a general type of entry vehicle. But they are function of the maximum lift-to-drag ratio $E^{*}$ as it should be, since $E^{*}$ is an important design parameter for vehicle performance.

The control laws can be expressed in terms of the state variables if the unknown adjoint vector $\mathbf{p}$ can be expressed in terms of these variables through integration of the adjoint equations

$$
\frac{\mathrm{d} p_{x}}{\mathrm{~d} h}=-\frac{\partial H}{\partial x}
$$

where $x$ is any one of the state variables.

In general, the five equations (17) are coupled with the five equations (11). These equations can be integrated analytically if we consider separately the flight in two widely different regions: an outer region, at high altitude, where aerodynamic force is weak compared with gravitational force, and an inner region, near the planetary surface where aerodynamic force is predominant. The inner and outer solutions are matched directly and a composite solution, valid everywhere, is constructed by additive composition.

\section{Outer expansions (Keplerian region)}

The outer expansions are introduced to study the limiting condition of the solution in the region near the vacuum where the gravitational force is predominant. They are obtained by repeated application of the outer limit, which is defined as the limit when $\epsilon \rightarrow 0$ with the variable $h$ and other dimensionless quantities held fixed.

We assume the following expansions

$$
\begin{aligned}
\alpha & =\alpha_{0}(h)+\epsilon \alpha_{1}(h)+\cdots \\
\Omega & =\Omega_{0}(h)+\epsilon \Omega_{1}(h)+\cdots \\
I & =I_{0}(h)+\epsilon I_{1}(h)+\cdots \\
u & =u_{0}(h)+\epsilon u_{1}(h)+\cdots \\
\gamma & =\gamma_{0}(h)+\epsilon \gamma_{1}(h)+\cdots
\end{aligned}
$$

By substituting into eqns (11) and equating coefficients of like power in $\epsilon$, the differential equations with zero order of $\epsilon$ are

$$
\begin{aligned}
& \frac{\mathrm{d} \alpha_{0}}{\mathrm{~d} h}=\frac{\cot \gamma_{0}}{1+h} \\
& \frac{\mathrm{d} \Omega_{0}}{\mathrm{~d} h}=0 \\
& \frac{\mathrm{d} I_{0}}{\mathrm{~d} h}=0 \\
& \frac{\mathrm{d} u_{0}}{\mathrm{~d} h}=-\frac{2}{(1+h)^{2}}
\end{aligned}
$$




$$
\frac{\mathrm{d} \gamma_{0}}{\mathrm{~d} h}=\left[\frac{1}{(1+h)}-\frac{1}{u_{0}(1+h)^{2}}\right] \cot \gamma_{0}
$$

The solution of the system is

$$
\begin{gathered}
\frac{u}{2}-\frac{1}{1+h}=c_{1} \\
\cos \left(\alpha-c_{3}\right)=\frac{c_{2}^{2}}{\sqrt{\left(2 c_{1} c_{2}{ }^{2}+1\right)(1+h)}}-\frac{1}{\sqrt{ }\left(2 c_{1} c_{2}{ }^{2}+1\right)} \\
\Omega=c_{4} \\
I=c_{5}
\end{gathered}
$$

where the $c_{j}$ are constants of integration.

Since we consider only first order solution, subscript zero has been omitted for convenience. The adjoint equations, eqns (17), in the outer region, are

$$
\begin{aligned}
\frac{\mathrm{d} p_{\alpha}}{\mathrm{d} h} & =0 \\
\frac{\mathrm{d} p_{\Omega}}{\mathrm{d} h} & =0 \\
\frac{\mathrm{d} p_{I}}{\mathrm{~d} h} & =0 \\
\frac{\mathrm{d} p_{u}}{\mathrm{~d} h} & =-\frac{p_{\gamma} \cot \gamma}{u^{2}(1+h)^{2}} \\
\frac{\mathrm{d} p_{\gamma}}{\mathrm{d} h} & =\left[\frac{p_{\alpha}}{1+h}+p_{\gamma}\left(\frac{1}{1+h}-\frac{1}{u(1+h)^{2}}\right)\right] \frac{1}{\sin ^{2} \gamma} .
\end{aligned}
$$

Upon using the solution (20) and integrating we have

$$
\begin{gathered}
p_{\alpha}=a_{1} \\
p_{\Omega}=a_{2} \\
p_{1}=a_{3} \\
p_{u}=-\frac{a_{5}}{2 u}+\frac{a_{1} c_{2} \sin \gamma}{\left(2 c_{1} c_{2}^{2}+1\right) u^{1 / 2}}+a_{4} \\
p_{\gamma}=a_{5} \tan \gamma-\frac{2 a_{1}\left[c_{1}(1+h)+c_{1} c_{2}^{2}+1\right]}{\left(2 c_{1} c_{2}{ }^{2}+1\right)}
\end{gathered}
$$

where the $a_{i}$ constitute another set of constants of integration providing a complete solution in the outer region.

\section{Inner expansions (aerodynamic-predominated region)}

The inner expansions are introduced to study the limiting condition of the solution near the planetary surface where the aerodynamic force is predominant. 
They are obtained by repeated application of the inner limit, which is defined as the limit when $\epsilon \rightarrow 0$ with the new stretched altitude

$$
\bar{h}=\frac{h}{\epsilon}
$$

and other dimensionless quantities held fixed.

We assume the following expansions

$$
\begin{aligned}
\alpha & =\bar{\alpha}_{0}(\bar{h})+\epsilon \bar{\alpha}_{1}(\bar{h})+\cdots \\
\Omega & =\bar{\Omega}_{0}(\bar{h})+\epsilon \bar{\Omega}_{1}(\bar{h})+\cdots \\
I & =\bar{I}_{0}(\bar{h})+\epsilon \bar{I}_{1}(\bar{h})+\cdots \\
u & =\bar{u}_{0}(\bar{h})+\epsilon \bar{u}_{1}(\bar{h})+\cdots \\
\gamma & =\bar{\gamma}_{0}(\bar{h})+\epsilon \bar{\gamma}_{1}(\bar{h})+\cdots
\end{aligned}
$$

By substituting into eqns (11) and equating coefficients of like powers in $\epsilon$, the differential equations with zero order of $\epsilon$ are

$$
\begin{aligned}
\frac{\mathrm{d} \bar{\alpha}_{0}}{\mathrm{~d} \bar{h}} & =-\frac{\sin \bar{\alpha}_{0}}{\tan \bar{I}_{0}}\left(\frac{B \lambda \sin \sigma \mathrm{e}^{-\bar{h}}}{\sin \bar{\gamma}_{0} \cos \bar{\gamma}_{0}}\right) \\
\frac{\mathrm{d} \bar{\Omega}_{0}}{\mathrm{~d} \bar{h}} & =\frac{\sin \bar{\alpha}_{0}}{\sin \bar{I}_{0}}\left(\frac{B \lambda \sin \sigma \mathrm{e}^{-\bar{h}}}{\sin \bar{\gamma}_{0} \cos \bar{\gamma}_{0}}\right) \\
\frac{\mathrm{d} \bar{I}_{0}}{\mathrm{~d} \bar{h}} & =\cos \bar{\alpha}_{0}\left(\frac{B \lambda \sin \sigma \mathrm{e}^{-\bar{h}}}{\sin \bar{\gamma}_{0} \cos \bar{\gamma}_{0}}\right) \\
\frac{\mathrm{d} \bar{u}_{0}}{\mathrm{~d} \bar{h}} & =-\frac{2 B f(\lambda) \bar{u}_{0} \mathrm{e}^{-\bar{h}}}{E^{*} \sin \bar{\gamma}_{0}} \\
\frac{\mathrm{d} \bar{\gamma}_{0}}{\mathrm{~d} \bar{h}} & =\frac{B \lambda \cos \sigma \mathrm{e}^{-\bar{h}}}{\sin \bar{\gamma}_{0}} .
\end{aligned}
$$

This set of equations has two first integrals

$$
\begin{gathered}
\sin \bar{\alpha}_{0} \sin \bar{I}_{0}=\sin \bar{c}_{3} \\
\cos \bar{\alpha}_{0}=\cos \bar{c}_{3} \cos \left(\bar{c}_{4}-\bar{\Omega}_{0}\right) .
\end{gathered}
$$

The integration of the other equations requires the knowledge of lift and bank control. For constant $\lambda$ and $\sigma$, the equations have been integrated completely (Busemann et al., 1975). Although complete solution for variable $\lambda$ and $\sigma$ does not seem possible, it is not necessary for obtaining the optimal control since we are only seeking the solution of the adjoint vector $p$.

The adjoint equations in the inner region are

$$
\begin{aligned}
\frac{\mathrm{d} \bar{p}_{\alpha}}{\mathrm{d} \bar{h}} & =\frac{B \lambda \sin \sigma \mathrm{e}^{-\bar{h}}}{\sin \bar{\gamma}_{0} \cos \bar{\gamma}_{0}}\left[\bar{p}_{\alpha} \frac{\cos \bar{\alpha}_{0}}{\tan \bar{I}_{0}}-\bar{p}_{\Omega} \frac{\cos \bar{\alpha}_{0}}{\sin \bar{I}_{0}}+\bar{p}_{I} \sin \bar{\alpha}_{0}\right] \\
\frac{\mathrm{d} \bar{p}_{\Omega}}{\mathrm{d} \bar{h}} & =0
\end{aligned}
$$




$$
\begin{aligned}
\frac{\mathrm{d} \bar{p}_{I}}{\mathrm{~d} \bar{h}}= & \frac{B \lambda \sin \sigma \mathrm{e}^{-\bar{h}}}{\sin \bar{\gamma}_{0} \cos \bar{\gamma}_{0}}\left[-\bar{p}_{\alpha} \frac{\sin \bar{\alpha}_{0}}{\sin ^{2} \bar{I}_{0}}+\bar{p}_{\Omega} \frac{\sin \bar{\alpha}_{0} \cos \bar{I}_{0}}{\sin ^{2} \bar{I}_{0}}\right] \\
\frac{\mathrm{d} \bar{p}_{u}}{\mathrm{~d} \bar{h}}= & \frac{2 \bar{p}_{u} B f(\lambda) \mathrm{e}^{-\bar{h}}}{E^{*} \sin \bar{\gamma}_{0}} \\
\frac{\mathrm{d} \bar{p}_{\gamma}}{\mathrm{d} \bar{h}}= & B \lambda \sin \sigma \mathrm{e}^{-\bar{h}}\left[-\bar{p}_{\alpha} \frac{\sin \bar{\alpha}_{0}}{\tan \bar{I}_{0}}+\bar{p}_{\Omega} \frac{\sin \bar{\alpha}_{0}}{\sin \bar{I}_{0}}+\bar{p}_{I} \cos \bar{\alpha}_{0}\right]\left(\frac{1}{\sin ^{2} \bar{\gamma}_{0}}-\frac{1}{\cos ^{2} \bar{\gamma}_{0}}\right) \\
& -\frac{2 \bar{p}_{u} B f(\lambda) \bar{u}_{0} \mathrm{e}^{-\bar{h}} \cos \bar{\gamma}_{0}}{E^{*} \sin ^{2} \bar{\gamma}_{0}}+\bar{p}_{\gamma} \frac{B \lambda \cos \sigma \mathrm{e}^{-\bar{h}} \cos \bar{\gamma}_{0}}{\sin ^{2} \bar{\gamma}_{0}} .
\end{aligned}
$$
that

By changing the independent variable from $\bar{h}$ to $\bar{\Omega}_{0}$, and integrating, it is found

$$
\begin{gathered}
\bar{p}_{\alpha}=\sin \bar{I}_{0}\left(\bar{a}_{3} \sin \bar{\Omega}_{0}-\bar{\alpha}_{1} \cos \bar{\Omega}_{0}\right)+\bar{a}_{2} \cos \bar{I}_{0} \\
\bar{p}_{\Omega}=\bar{a}_{2} \\
\bar{p}_{I}=\bar{a}_{1} \sin \bar{\Omega}_{0}+\bar{a}_{3} \cos \bar{\Omega}_{0} .
\end{gathered}
$$

Next, it is seen that

$$
\bar{p}_{u}=\frac{\bar{a}_{4}}{\bar{u}_{0}}
$$

where the $\bar{a}_{\mathrm{j}}$ are constants of integration.

To integrate the equation for $\bar{p}_{\gamma}$ we observe by direct verification that the following quantity

$$
K=-\bar{p}_{\alpha} \frac{\sin \bar{\alpha}_{0}}{\tan \bar{I}_{0}}+\bar{p}_{\Omega} \frac{\sin \bar{\alpha}_{0}}{\sin \bar{I}_{0}}+\bar{p}_{I} \cos \bar{\alpha}_{0}
$$

is constant in the inner region. Then we write the solution for variable lift and bank control, eqns (15) and (16), in the inner region

$$
\tan \sigma=\frac{K}{\bar{p}_{\gamma} \cos \bar{\gamma}_{0}}, \quad \cos \sigma=\frac{E^{*} \bar{p}_{\gamma}}{2 \bar{p}_{u} \bar{u}_{0} f^{\prime}(\lambda)}
$$

where $f^{\prime}=\mathrm{d} f / \mathrm{d} \lambda$. Equation for $\bar{p}_{\gamma}$, when changed to $\bar{\gamma}_{0}$ as independent variable, becomes

$$
\frac{\mathrm{d} \bar{p}_{\gamma}}{\mathrm{d} \bar{\gamma}_{0}}=K \tan \sigma\left(\frac{1}{\sin \bar{\gamma}_{0}}-\frac{\sin \bar{\gamma}_{0}}{\cos ^{2} \bar{\gamma}_{0}}\right)-\frac{2 \bar{p}_{u} \bar{u}_{0} f(\lambda) \cos \bar{\gamma}_{0}}{E^{*} \lambda \sin \sigma \sin \bar{\gamma}_{0}}+\bar{p}_{\gamma} \cot \bar{\gamma}_{0}
$$

With the solutions (29) and (31), we write

$$
\frac{\mathrm{d} \bar{p}_{\gamma}}{\mathrm{d} \bar{\gamma}_{0}}=\frac{K^{2}}{\bar{p}_{\gamma} \cos \bar{\gamma}_{0}}\left(\frac{1}{\sin \bar{\gamma}_{0}}-\frac{\sin \bar{\gamma}_{0}}{\cos ^{2} \bar{\gamma}_{0}}\right)-\frac{4 \bar{a}_{4}^{2} f(\lambda) f^{\prime}(\lambda)}{E^{*^{2}} \lambda \bar{p}_{\gamma}} \cot \bar{\gamma}_{0}+\bar{p}_{\gamma} \cot \bar{\gamma}_{0}
$$


Let

$$
\Gamma=\frac{K^{2}}{\cos ^{2} \bar{\gamma}_{0}}+\bar{p}_{\gamma}^{2}
$$

Using $\Gamma$ to replace $\bar{p}_{\gamma}$ we have a linear equation

$$
\frac{\mathrm{d} \Gamma}{\mathrm{d} \bar{\gamma}_{0}}=\frac{2 \cos \bar{\gamma}_{0}}{\sin \bar{\gamma}_{0}} \Gamma-\frac{8 \bar{a}_{4}{ }^{2} f(\lambda) f^{\prime}(\lambda)}{E^{*^{2}} \lambda} \cot \bar{\gamma}_{0} .
$$

From eqns (15) and (16) we notice that

$$
\left[f^{\prime}(\lambda)\right]^{2}=\frac{E^{* 2}}{4 \bar{a}_{4}^{2}}\left[\frac{K^{2}}{\cos ^{2} \bar{\gamma}_{0}}+\bar{p}_{\gamma}^{2}\right]=\frac{E^{* 2} \Gamma}{4 \bar{a}_{4}^{2}} .
$$

On the other hand, we write eqn (35)

$$
\frac{\mathrm{d} \Gamma}{\mathrm{d} \bar{\gamma}_{0}}=2 \cot \bar{\gamma}_{0}\left[\Gamma-\frac{4 \bar{a}_{4}^{2} f(\lambda) f^{\prime}(\lambda)}{E^{* 2} \lambda}\right] .
$$

If the drag polar function $f(\lambda)$ is known, from eqn (36), $\lambda$ can be solved in terms of $\Gamma$ and upon substituting into eqn (37), $\Gamma$ is obtained by simple quadrature.

We consider a generalized drag polar of the form

$$
f(\lambda)=\frac{(n-1)+\lambda^{n}}{n}
$$

with of course $n=2$ for parabolic polar.

Then

$$
\Gamma=\frac{4 \bar{a}_{4}^{2}}{E^{* 2}} \lambda^{2(n-1)}
$$

and eqn (37) has the form

$$
\frac{\mathrm{d} \lambda^{n}}{\mathrm{~d} \bar{\gamma}_{0}}=\left(\lambda^{n}-1\right) \cot \bar{\gamma}_{0}
$$

Upon integration

$$
\lambda^{n}=1+\bar{a}_{5} \sin \bar{\gamma}_{0}
$$

where $\bar{a}_{5}$ is the last constant of integration for the adjoint system. The solution for $p_{\gamma}$ is then

$$
\bar{p}_{\gamma}^{2}=\frac{4 \bar{a}_{4}^{2}}{E^{* 2}}\left(1+\bar{a}_{5} \sin \bar{\gamma}_{0}\right)^{[2(n-1) / n]}-\frac{K^{2}}{\cos ^{2} \bar{\gamma}_{0}}
$$


It seems surprising that the solution (41) for the lift control is the same as for the free range problem (Contensou, 1965). The reason for this is that, by using the orbital elements as variables we obtain the integrals (26) which, upon identifying with relations (10), show that the positions $\theta$ and $\phi$ remain constant in the inner region. Also, it should be noted that $K$ is not a new constant of integration. In the inner region, it is simply a combination of existing constants of integration.

\section{Asymptotic matching and composite solution}

The constants of integration $\bar{a}_{j}$ in the inner expansions will be determined by matching with the outer expansions. In this problem, matching will be accomplished by expanding the inner solutions for large $\bar{h}$, expressing the results in terms of the outer variables and matching with the outer solutions for small $h$.

The outer state variables, eqns (20), become for small $h$

$$
\begin{gathered}
u=2\left(c_{1}+1\right) \\
\cos \gamma=\frac{c_{2}}{\sqrt{ }\left[2\left(c_{1}+1\right)\right]} \\
\cos \left(\alpha-c_{3}\right)=\frac{c_{2}{ }^{2}-1}{\sqrt{ }\left(2 c_{1} c_{2}{ }^{2}+1\right)} \\
\Omega=c_{4} \\
I=c_{5} .
\end{gathered}
$$

The outer adjoint variables, eqns (22), become for small $h$

$$
\begin{aligned}
& p_{\alpha}=a_{1} \\
& p_{\Omega}=a_{2} \\
& p_{I}=a_{3} \\
& p_{u}=a_{4}-\frac{a_{5}}{4\left(c_{1}+1\right)} \pm \frac{a_{1} c_{2} \sqrt{ }\left[2\left(c_{1}+1\right)-c_{2}^{2}\right]}{2\left(c_{1}+1\right)\left(2 c_{1} c_{2}^{2}+1\right)} \\
& p_{\gamma}= \pm \frac{a_{5} \sqrt{ }\left[2\left(c_{1}+1\right)-c_{2}^{2}\right]}{c_{2}}-\frac{2 a_{1}\left(c_{1}+c_{1} c_{2}^{2}+1\right)}{\left(2 c_{1} c_{2}^{2}+1\right)}
\end{aligned}
$$

The upper sign ( \pm ) correspond to positive $\sin \gamma$. On the other hand, the inner adjoint variables, eqns (28), (29) and (42), become for large $\bar{h}$

$$
\begin{aligned}
& \bar{p}_{\alpha}=\sin c_{5}\left(\bar{a}_{3} \sin c_{4}-\bar{a}_{1} \cos c_{4}\right)+\bar{a}_{2} \cos c_{5} \\
& \bar{p}_{\Omega}=\bar{a}_{2} \\
& \bar{p}_{I}=\bar{a}_{1} \sin c_{4}+\bar{a}_{3} \cos c_{4} \\
& \bar{p}_{u}=\frac{\bar{a}_{4}}{2\left(c_{1}+1\right)} \\
& \tilde{p}_{\gamma}=\left[\frac { 4 \overline { a } _ { 4 } ^ { 2 } } { E ^ { * ^ { 2 } } } \left(1 \pm \bar{a}_{5} \sqrt{\left.\left.\left[\frac{2\left(c_{1}+1\right)-c_{2}{ }^{2}}{2\left(c_{1}+1\right)}\right]\right)^{2(n-1) / n}-\frac{2 \bar{K}^{2}\left(c_{1}+1\right)}{c_{2}{ }^{2}}\right]^{1 / 2}}\right.\right.
\end{aligned}
$$

where $\bar{K}$ is the limit of $K$ for large $\bar{h}$.

By equating the two sets of equations (44) and (45) by pair we can evaluate the 
constants of integrations $\bar{a}_{j}$, in terms of the constants of integration $a_{j}$ and $c_{j}$.

The composite expansions, uniformly valid everywhere, can be constructed by the method of additive composition. The additive composition is obtained by taking the sum of the inner and the outer expansions and subtracting the part they have in common.

For $p_{\alpha}, p_{\Omega}$ and $p_{I}$, it is clear that they are identical to the inner solution, so that instead of using the constants $a_{j}$, we can write the composite solution with the constant $\bar{a}_{j}$, and we have

$$
\begin{gathered}
p_{\alpha c}=\sin I\left(\bar{a}_{3} \sin \Omega-\bar{a}_{1} \sin \Omega\right)+\bar{a}_{2} \cos I \\
p_{\Omega c}=\bar{a}_{2} \\
p_{\text {Ic }}=\bar{a}_{1} \sin \Omega+\bar{a}_{3} \cos \Omega .
\end{gathered}
$$

It is easy to verify, using the exact equations, that this solution is exact. In this case, the method has a perfect matching. The solutions for $p_{u c}$ and $p_{\gamma c}$ are only correct to the zero order of $\epsilon$.

For $p_{u c}$, using the method of additive composition, we have

$$
p_{u c}=p_{u}+\bar{p}_{u}-\frac{\bar{a}_{4}}{2\left(c_{1}+1\right)}
$$

where $p_{u}$ is the outer solution, expressed in outer variables, and $\bar{p}_{u}$ is the inner solution, expressed in inner variables. Fortunately all the outer solutions can be expressed in terms of the independent variable $h$, so that we can have the composite solution expressed explicitly in terms of the inner variables alone as was the case of eqn (46). Hence, expressing $p_{u}$ in terms of $h$, we have

$$
p_{u c}=\frac{\bar{a}_{4}}{u}-\frac{\bar{a}_{4}}{2\left(c_{1}+1\right)}+a_{4}-\frac{a_{5}(1+h)}{4\left[c_{1}(1+h)+1\right]} \pm \frac{a_{1} c_{2} V\left[2 c_{1}(1+h)^{2}+2(1+h)-c_{2}^{2}\right]}{2\left(2 c_{1} c_{2}^{2}+1\right)\left[c_{1}(1+h)+1\right]} .
$$

Similarly, we have for $p_{\gamma_{t}}$

$$
\begin{aligned}
\bar{p}_{\gamma c}= & \pm \frac{a_{5}}{c_{2}} \sqrt{ }\left[2 c_{1}(1+h)^{2}+2(1+h)-c_{2}^{2}\right]-\frac{2 a_{1}\left[c_{1}(1+h)+c_{1} c_{2}^{2}+1\right]}{\left(2 c_{1} c_{2}{ }^{2}+1\right)} \\
& +\left[\frac{4 \bar{a}_{4}^{2}}{E^{* 2}}\left(1+\bar{a}_{5} \sin \gamma\right)^{2(n-1) / n}-\frac{K^{2}}{\cos ^{2} \gamma}\right]^{1 / 2} \\
& \mp \frac{a_{5} \sqrt{ }\left[2\left(c_{1}+1\right)-c_{2}^{2}\right]}{c_{2}}+\frac{2 a_{1}\left(c_{1}+c_{1} c_{2}{ }^{2}+1\right)}{\left(2 c_{1} c_{2}^{2}+1\right)} .
\end{aligned}
$$

Using the composite solution, eqns (46)-(48) in the eqns (15) and (16) we have the optimal control for variable lift and bank, uniformly valid everywhere in terms of the state variables $\alpha, \Omega, I, u$ and $\gamma$, of the independent variable $h$, and a mixed set of constants of integration.

It has been shown in the past (Vinh et al., 1975) that, under certain simplifying assumptions, the adjoint equations can be integrated separately and hence, the 
solution for optimal aerodynamic controls contains 5 independent constants of integration. Since theoretically the adjoints equations are coupled with the state equations some dependence of the adjoint solutions on the initial state variables must exist. It is displayed in the composite solutions for $p_{u}$ and $p_{\gamma}$, eqns (47) and (48).

For practical applications, the constants of integration $\bar{a}_{1}, \bar{a}_{2}$ and $\bar{a}_{3}$ in the exact solutions, eqns (46), are considered as arbitrary and to be determined by the appropriate transversality conditions.

The expression $K$ in the composite solution for $p_{\gamma}$, eqn (48), is no longer a constant but it is varying according to

$$
K=-p_{a c} \frac{\sin \alpha}{\tan I}+p_{\Omega c} \frac{\sin \alpha}{\sin I}+p_{I c} \cos \alpha
$$

where the adjoints $p_{\alpha c}, p_{\Omega c}$ and $p_{I c}$ are given by eqn (46).

The constants $c_{1}$ and $c_{2}$ in the solutions (47) and (48) can be evaluated by using the outer solutions for $u$ and $\gamma$, eqns (20), applied at the initial conditions. Hence

$$
\begin{aligned}
& c_{1}=\frac{u_{i}}{2}-\frac{1}{1+h_{i}} \\
& c_{2}=\left(1+h_{i}\right) u_{i}^{1 / 2} \cos \gamma_{i}
\end{aligned}
$$

where subscript $i$ denotes the initial conditions.

The constants $a_{1}, a_{2}$ and $a_{3}$ are obtained by matching the inner and outer solutions for $p_{\alpha}, p_{\Omega}$ and $p_{I}$.

$$
\begin{aligned}
& a_{1}=\sin I_{i}\left(\bar{a}_{3} \sin \Omega_{i}-\bar{a}_{1} \cos \Omega_{i}\right)+\bar{a}_{2} \cos I_{i} \\
& a_{2}=\bar{a}_{2} \\
& a_{3}=\bar{a}_{1} \sin \Omega_{i}+\bar{a}_{3} \cos \Omega_{i \cdot} .
\end{aligned}
$$

Finally, either set $\left(a_{4}, a_{5}\right)$ or $\left(\bar{a}_{4}, \bar{a}_{5}\right)$ can be used as the remaining independent constants of integration. They are related by matching the solutions for $p_{u}$ and $p_{\gamma}$. We have the relations

$$
\frac{\bar{a}_{4}}{2\left(c_{1}+1\right)}=a_{4}-\frac{a_{5}}{4\left(c_{1}+1\right)} \pm \frac{a_{1} c_{2} V\left[2\left(c_{1}+1\right)-c_{2}^{2}\right]}{2\left(c_{1}+1\right)\left(2 c_{1} c_{2}^{2}+1\right)}
$$

and

$$
\begin{aligned}
& \pm \frac{a_{5} V\left[2\left(c_{1}+1\right)-c_{2}^{2}\right]}{c_{2}}-\frac{2 a_{1}\left(c_{1}+c_{1} c_{2}^{2}+1\right)}{\left(2 c_{1} c_{2}{ }^{2}+1\right)} \\
& \quad=\left[\frac { 4 \overline { a } _ { 4 } ^ { 2 } } { E ^ { * ^ { 2 } } } \left(1 \pm \bar{a}_{5} \sqrt{\left.\left.\left(\frac{2\left(c_{1}+1\right)-c_{2}{ }^{2}}{2\left(c_{1}+1\right)}\right)\right)^{2(n-1) / n}-\frac{2 \bar{K}^{2}\left(c_{1}+1\right)}{c_{2}{ }^{2}}\right]^{1 / 2}}\right.\right.
\end{aligned}
$$

where the constant $\bar{K}$ is the limit of $K$ for large $\bar{h}$, that is

$$
\bar{K}=a_{1} \frac{\sin \alpha}{\tan I_{i}}+a_{2} \frac{\sin \alpha}{\sin I_{i}}+a_{3} \cos \alpha
$$


The limiting constant value $\alpha$ is provided by

$$
\cos \left(\alpha-c_{3}\right)=\frac{c_{2}^{2}-1}{\sqrt{\left(2 c_{1} c_{2}^{2}+1\right)}}
$$

with $c_{3}$ obtained from the initial conditions applied to the outer solution

$$
\cos \left(\alpha_{i}-c_{3}\right)=\frac{c_{2}^{2}}{\sqrt{ }\left(2 c_{1} c_{2}^{2}+1\right)\left(1+h_{i}\right)}-\frac{1}{\sqrt{ }\left(2 c_{1} c_{2}^{2}+1\right)}
$$

In summary, the optimal solution contains 5 arbitrary constants of integration $\bar{a}_{i}$, and depends on the five initial conditions $\alpha_{i}, \Omega_{i}, I_{i}, u_{i}$ and $\gamma_{i}$.

A check of the computational steps presented above will convince the reader that the $a_{i}$ and the $c_{i}$ can be expressed explicitly in terms of the $\bar{a}_{i}$ and the $x_{i}$ for any arbitrary $n$. Hence, although somewhat cumbersome, the optimal solution can be expressed explicitly in terms of five constants of integration $\bar{a}_{i}$, of the five state variables $\alpha, \Omega, I, u, \gamma$ and their initial values, and the independent variable $h$.

\section{References}

Busemann, A., Vinh, N. X. and Culp, R. D. (1975) Solution of the exact equations for three-dimensional atmospheric entry using directly matched asymptotic expansions, NASA Report, to appear.

Contensou, P. (1965) Contribution à l'étude schématique des trajectoires semi-ballistique à grande portée, Communication to Association Technique Maritime et Aéronautique, Paris.

Shi, Y. Y. (1971) Matched asymptotic solutions for optimum lift controlled atmospheric entry, AIAA J. 9, $2229-2238$.

Vinh, N. X., Busemann, A. and Culp, R. D. (1975) Optimum three-dimensional atmospheric entry, Acta Astronautica 2, 7/8, 593-611. 